\title{
An Adaptive Variable Order Quadrature Strategy
}

\author{
Paul Houston and Thomas P. Wihler
}

\begin{abstract}
We propose a new adaptive numerical quadrature procedure which includes both local subdivision of the integration domain, as well as local variation of the number of quadrature points employed on each subinterval. In this way we aim to account for local smoothness properties of the integrand as effectively as possible, and thereby achieve highly accurate results in a very efficient manner. Indeed, this idea originates from so-called $h p$-version finite element methods which are known to deliver high-order convergence rates, even for nonsmooth functions.
\end{abstract}

\section{Introduction}

Numerical integration methods have witnessed a tremendous development over the last few decades; see, e.g., [2, 3, 14]. In particular, adaptive quadrature rules have nowadays become an integral part of many scientific computing codes. Here, one of the first yet very successful approaches is the application of adaptive Simpson integration or the more accurate Gauss-Kronrod procedures (see, e.g., [7]). The key points in the design of these methods are, first of all, to keep the number of function evaluations low, and, secondly, to divide the domain of integration in such a way that the features of the integrand function are appropriately and effectively accounted for.

The aim of the current article is to propose a complementary adaptive quadrature approach that is quite different from previous numerical integration schemes. In fact, our work is based on exploiting ideas from $h p$-type adaptive finite element

Paul Houston

School of Mathematical Sciences, University of Nottingham, University Park, Nottingham, NG7

2RD, UK, e-mail: Paul.Houston@ nottingham.ac.uk

Thomas P. Wihler

Mathematisches Institut, Universität Bern, Sidlerstrasse 5, CH-3012 Bern, Switzerland, e-mail: wihler@math.unibe.ch. TW acknowledges the financial support by the Swiss National Science Foundation (SNF) 
methods (FEM); cf. [4, 6, 11, 12, 20]. These schemes accommodate and combine both traditional low-order adaptive FEM and high-order (so-called spectral) methods within a single unified framework. Specifically, their goal is to generate discrete approximation spaces which allow for both adaptively refined subdomains, as well as locally varying approximation orders. In this way, the $h p$-FEM methodology is able to resolve features of an underlying unknown analytical solution in a highly efficient manner. In fact, this approach has proved to be enormously successful in the context of numerically approximating solutions of differential equations, and has been shown to exhibit high-order algebraic or exponential convergence rates even in the presence of local singularities; cf. $[8,16,18]$.

With this in mind, we adopt the $h p$-adaptive FEM strategy for the purpose of introducing a variable order adaptive quadrature framework. More precisely, we propose a procedure whereby the integration domain will be subdivided adaptively in combination with a local tuning of the number of quadrature points employed on each subinterval. To drive this refinement process, we employ a smoothness estimation technique from $[6,21]$ (see also [11] for a related strategy), which was originally introduced in the context of $h p$-adaptive FEMs. Specifically, the smoothness test makes it possible to gain local information concerning the regularity of the integrand function, and thereby, to suitably subdivide the integration domain and select an appropriate number of quadrature points for each subinterval. By means of a series of numerical experiments we demonstrate that the proposed adaptive quadrature strategy is capable of generating highly accurate approximations at a very low computational cost. The main ideas on this new approach together with a view on practical aspects will be discussed in the subsequent section.

\section{An $h p$-Type Quadrature Approach}

Typical quadrature rules for the approximation of an integral $I:=\int_{-1}^{1} f(x) \mathrm{d} x$ of a continuous function $f:[-1,1] \rightarrow \mathbb{R}$, take the form $I \approx \widehat{Q}_{p}(f):=\sum_{k=1}^{p} w_{p, k} f\left(\widehat{x}_{p, k}\right)$, where $p \geq 1$ is a (typically prescribed) integer number, and $\left\{\widehat{x}_{p, k}\right\}_{k=1}^{p} \subset[-1,1]$ and $\left\{w_{p, k}\right\}_{k=1}^{p} \subset(0,2]$ are appropriate quadrature points and weights, respectively. When dealing with a variable number $p$ of quadrature points and weights, we can consider one-parameter families of quadrature rules (such as, for example, Gausstype quadrature methods); here, for each $p \in \mathbb{N}$, with $p \geq p_{\min }$, where $p_{\min }$ is a minimal number of points, there are (possibly non-hierarchical) families of quadrature points $\widehat{\boldsymbol{x}}_{p}=\left\{\widehat{x}_{p, k}\right\}_{k=1}^{p}$, and weights $\boldsymbol{w}_{p}=\left\{w_{p, k}\right\}_{k=1}^{p}$.

On an arbitrary bounded interval $[a, b], a<b$, a corresponding integration formula can be obtained, for instance, by means of a simple affine scaling $\phi_{[a, b]}$ : $[-1,1] \rightarrow[a, b], \widehat{x} \mapsto x=\phi_{[a, b]}(\widehat{x})=\frac{1}{2} h \widehat{x}+\frac{1}{2}(a+b)$, with $h=b-a>0$. Indeed, in this case $\int_{a}^{b} f(x) \mathrm{d} x \approx Q_{[a, b], p}(f):=h / 2 \sum_{k=1}^{p} w_{p, k}\left(f \circ \phi_{[a, b]}\right)\left(\widehat{x}_{p, k}\right)$, where $f:[a, b] \rightarrow$ $\mathbb{R}$ is again continuous. As before, for any specific family of quadrature rules, the 
corresponding quadrature point families $\boldsymbol{x}_{p}$ are obtained in a straightforward way by letting $\boldsymbol{x}_{p}=\phi_{[a, b]}\left(\widehat{\boldsymbol{x}}_{p}\right)$ (noting that $\phi_{[a, b]}$ is extended componentwise to vectors).

Furthermore, the above construction allows us to define composite quadrature rules, whereby the integral of $f$ is approximated on a collection of $n \geq 1$ disjoint (open) subintervals $\left\{K_{i}\right\}_{i=1}^{n}$ of $[a, b]$ with $[a, b]=\bigcup_{i=1}^{n} \bar{K}_{i}$, i.e., $I \approx \sum_{i=1}^{n} Q_{K_{i}, p}\left(\left.f\right|_{K_{i}}\right)$.

\subsection{The Basic Idea: hp-Adaptivity}

Adaptive quadrature rules usually generate a sequence of repeatedly bisected and possibly non-uniform subintervals $\left\{K_{i}\right\}_{i=1}^{n}, n \geq 1$, of the integration domain $[a, b]$ (i.e., each subinterval $K_{i}$ may have a different length $h_{i}$ ), with a prescribed and uniform number $p$ of quadrature points on each subinterval. With the aim of providing highly accurate approximations with as little computational effort as possible, the novelty of the approach presented in this article is to design an adaptive quadrature procedure, which, in addition to subdividing the original interval $[a, b]$ into appropriate subintervals, is able to adjust the number of quadrature points $p_{i}$ individually within each subinterval $K_{i}$ in an effective way. We note that this idea originates from approximation theory $[5,15]$ (see also [8]), and has been applied with huge success in the context of FEMs for the numerical approximation of differential equations. Indeed, under certain conditions, the judicious combination of subinterval refinements ( $h$-refinement) and selection of local approximation orders ( $p$-refinement), which results in the class of so-called $h p$-FEMs, is able to achieve high-order algebraic or exponential rates of convergence, even for solutions with local singularities; see, e.g. [18]. In an effort to automate the combined $h$ - and $p$-refinement process, a number of $h p$-adaptive FEM approaches have been proposed in the literature; see, e.g, [13] and the references cited therein. In the current article, we pursue the smoothness estimation approach developed in [6,21] (cf. also [11]), and translate the idea into the context of adaptive variable order numerical quadrature.

Given a subinterval $K_{i}$ with $p_{i}$ quadrature points, we are given a current approximation $Q_{K_{i}, p_{i}}\left(\left.f\right|_{K_{i}}\right)$ of the subintegral $\int_{K_{i}} f(x) \mathrm{d} x \approx Q_{K_{i}, p_{i}}\left(\left.f\right|_{K_{i}}\right)$. Then, with the aim of improving the approximate value $Q_{K_{i}, p_{i}}\left(\left.f\right|_{K_{i}}\right)$, in the sense of an $h p$-adaptive FEM methodology in one-dimension, we propose two possible refinements of $K_{i}$ :

(i) $h$-refinement: The subinterval $K_{i}$ of length $h_{i}$ is bisected into two subintervals $K_{i}^{1}$ and $K_{i}^{2}$ of equal size $h_{i} / 2$, and the number $p_{i}$ of quadrature points is either inherited to both subintervals or, in order to allow for derefinement with respect to the number of local quadrature points, reduced to $p_{i}-1$ points. In the latter case, we obtain the potentially improved approximation:

$$
Q_{K_{i}}^{\mathrm{h}}(f)=Q_{K_{i}^{1}, \max \left(1, p_{i}-1\right)}(f)+Q_{K_{i}^{2}, \max \left(1, p_{i}-1\right)}(f) .
$$

(ii) $p$-refinement: The subinterval $K_{i}$ is retained, and the number $p_{i}$ of quadrature points $p_{i}$ is increased by 1 , i.e., $p_{i} \leftarrow p_{i}+1$. This yields an approximation 


$$
Q_{K_{i}}^{\mathrm{p}}(f)=Q_{K_{i}, p_{i}+1}(f) .
$$

In case that $p_{i}=p_{\max }$, where $p_{\max }$ is a prescribed maximal number of quadrature points on each subinterval, we define

$$
Q_{K_{i}}^{\mathrm{p}}(f)=Q_{K_{i}^{1}, p_{i}}(f)+Q_{K_{i}^{2}, p_{i}}(f),
$$

where $K_{i}^{1}$ and $K_{i}^{2}$ result from subdividing $K_{i}$ as in (i).

In order to determine which of the above refinements is more appropriate for a given subinterval $K_{i}$, we apply a smoothness estimation idea outlined below. Once a decision between $h$ - and $p$-refinement for $K_{i}$ has been made, the procedure is repeated iteratively for any subintervals $K_{i}$ for which $Q_{K_{i}, p_{i}}\left(\left.f\right|_{K_{i}}\right)$ and its refined value (resulting from the chosen refinement) differ by at least a prescribed tolerance tol $>0$.

\subsection{Smoothness Estimation}

The basic idea presented in the articles $[6,11,21]$ is to estimate the regularity of a function to be approximated locally. Then, following along the lines of the $h p$ approximation approach, if the function is found to be smooth, according to the underlying regularity estimation test, then a $p$-refinement is performed, otherwise an $h$-refinement is employed. In [6], the following smoothness indicator, for a (weakly) differentiable function $f$ on an interval $K_{j}$, has been introduced (cf. [6, Eq. (3)]):

$$
\mathscr{F}_{K_{j}}[f]:= \begin{cases}\frac{\|f\|_{L^{\infty}\left(K_{j}\right)}}{h_{j}^{-1 / 2}\|f\|_{L^{2}\left(K_{j}\right)}+\frac{1}{\sqrt{2}} h_{j}^{1 / 2}\left\|f^{\prime}\right\|_{L^{2}\left(K_{j}\right)}} & \text { if }\left.f\right|_{K_{j}} \not \equiv 0, \\ 1 & \text { if }\left.f\right|_{K_{j}} \equiv 0 .\end{cases}
$$

The motivation behind this definition is the well-known continuous Sobolev embedding $W^{1,2}\left(K_{j}\right) \hookrightarrow L^{\infty}\left(K_{j}\right)$, which implies that $\mathscr{F}_{K_{j}}[f] \leq 1$ in (F); see [6, Proposition 1]. We classify $f$ as being smooth on $K_{j}$ if $\mathscr{F}_{K_{j}}[f] \geq \tau$, for a prescribed smoothness testing parameter $0<\tau<1$, and nonsmooth otherwise.

To begin, we first consider the special case when $f$ is a polynomial of degree $p_{j} \geq$ 1. Then, the derivative $f^{\left(p_{j}-1\right)}$ of order $p_{j}-1$ of $f$ is a linear polynomial, and the evaluation of the smoothness indicator $\mathscr{F}_{K_{j}}\left[f^{\left(p_{j}-1\right)}\right]$ from (F) is simple to obtain. In fact, let us write $\left.f\right|_{K_{j}}$ in terms of a (finite) Legendre series, that is,

$$
\left.f\right|_{K_{j}}=\sum_{l=0}^{p_{j}} a_{l}\left(\widehat{L}_{l} \circ \phi_{K_{j}}^{-1}\right)
$$

for coefficients $a_{0}, \ldots, a_{p_{j}} \in \mathbb{R}$. Here, $\widehat{L}_{l}, l \geq 0$, are the Legendre polynomials on $[-1,1]$ (scaled such that $\widehat{L}_{l}(1)=1$ for all $l \geq 0$ ), and $\phi_{K_{j}}$ is the affine scaling 
of $[-1,1]$ to $K_{j}$. For $f$ as in (4) it can be shown that

$$
\mathscr{F}_{K_{j}}\left[f^{\left(p_{j}-1\right)}\right]=\frac{1+\xi_{p_{j}}}{\sqrt{1+\frac{1}{3} \xi_{p_{j}}^{2}}+\sqrt{2} \xi_{p_{j}}}
$$

where $\xi_{p_{j}}=\left(2 p_{j}-1\right)\left|a_{p_{j}} / a_{p_{j}-1}\right|$ (provided that $a_{p_{j}-1} \neq 0$ ); see [6, Proposition 3]. In particular, this implies that, cf. [6, §2.2],

$$
\frac{1}{2} \approx \frac{\sqrt{3}}{\sqrt{6}+1} \leq \mathscr{F}_{K_{j}}\left[f^{\left(p_{j}-1\right)}\right] \leq 1
$$

In the context of the numerical integration rule, the above methodology can be adopted as follows: suppose we are given $p_{j} \geq 2$ quadrature points and weights, $\left\{\widehat{x}_{p_{j}, k}\right\}_{k=1}^{p_{j}}$ and $\left\{w_{p_{j}, k}\right\}_{k=1}^{p_{j}}$, respectively. Then,

$$
\int_{K_{j}} f(x) \mathrm{d} x \approx Q_{K_{j}, p_{j}}\left(\left.f\right|_{K_{j}}\right)=\frac{h_{j}}{2} \sum_{k=1}^{p_{j}} w_{p_{j}, k}\left(f \circ \phi_{K_{j}}\right)\left(\widehat{x}_{p_{j}, k}\right) .
$$

We denote the uniquely defined interpolating polynomial of $f$ of degree $p_{j}-1$ at the given quadrature points by $\Pi_{K_{j}, p_{j}-1} f=\sum_{l=0}^{p_{j}-1} b_{l}\left(\widehat{L}_{l} \circ \phi_{K_{j}}^{-1}\right)$. Due to orthogonality of the Legendre polynomials, we note that

$$
b_{l}=\frac{2 l+1}{h_{j}} \int_{K_{j}} \Pi_{K_{j}, p_{j}-1} f(x)\left(\widehat{L_{l}} \circ \phi_{K_{j}}^{-1}\right)(x) \mathrm{d} x, \quad l=0, \ldots, p_{j}-1 .
$$

We further assume that the quadrature rule under consideration is exact for all polynomials of degree up to $2 p_{j}-2$. Thereby,

$$
\begin{aligned}
b_{l} & =\frac{2 l+1}{2} \sum_{k=1}^{p_{j}} w_{p_{j}, k}\left(\Pi_{K_{j}, p_{j}-1} f\right) \circ \phi_{K_{j}}\left(\widehat{x}_{p_{j}, k}\right) \widehat{L}_{l}\left(\widehat{x}_{p_{j}, k}\right) \\
& =\frac{2 l+1}{2} \sum_{k=1}^{p_{j}} w_{p_{j}, k}\left(f \circ \phi_{K_{j}}\right)\left(\widehat{x}_{p_{j}, k}\right) \widehat{L}_{l}\left(\widehat{x}_{p_{j}, k}\right) .
\end{aligned}
$$

Consequently, we infer that

$$
\xi_{K_{j}, p_{j}-1}:=\left(2 p_{j}-3\right)\left|\frac{b_{p_{j}-1}}{b_{p_{j}-2}}\right|=\left(2 p_{j}-1\right) \frac{\sum_{k=1}^{p_{j}} w_{p_{j}, k}\left(f \circ \phi_{K_{j}}\right)\left(\widehat{x}_{p_{j}, k}\right) \widehat{L}_{p_{j}-1}\left(\widehat{x}_{p_{j}, k}\right)}{\sum_{k=1}^{p_{j}} w_{p_{j}, k}\left(f \circ \phi_{K_{j}}\right)\left(\widehat{x}_{p_{j}, k}\right) \widehat{L}_{p_{j}-2}\left(\widehat{x}_{p_{j}, k}\right)},
$$

and thus, in view of (5), we use the quantity

$$
\mathrm{F}_{K_{j}, p_{j}}(f):=\frac{1+\xi_{K_{j}, p_{j}-1}}{\sqrt{1+\frac{1}{3} \xi_{K_{j}, p_{j}-1}^{2}}+\sqrt{2} \xi_{K_{j}, p_{j}-1}} \in\left(\frac{\sqrt{3}}{\sqrt{6}+1}, 1\right)
$$


cf. (6), to estimate the smoothness of $\left.f\right|_{K_{j}}$. We note that the computation of $\xi_{K_{j}, p_{j}-1}$ does not require any additional function evaluations of $f$ since the values $(f \circ$ $\left.\phi_{K_{j}}\right)\left(\widehat{x}_{p_{j}, k}\right), k=1, \ldots, p_{j}$, have already been determined in the application of (7).

\subsection{Adaptive Variable Order Procedure}

Based on the above derivations, we propose an $h p$-type adaptive quadrature method. To this end, we start by choosing a tolerance tol $>0$, a smoothness parameter $\tau \in(\sqrt{3} /(\sqrt{6}+1), 1)$, and a maximal number $p_{\max } \geq 2$ of possible quadrature points on each subinterval. Furthermore, we define the interval $K_{1}=[a, b]$, and a small number $p_{1}, 2 \leq p_{1} \leq p_{\max }$, of quadrature points on $K_{1}$. Moreover, we initialise the set of subintervals subs, the order vector $p$ containing the number of quadrature points on each subinterval, and the unknown value $Q$ of the integral as follows: subs $=\left\{K_{1}\right\}, \mathrm{p}=\left\{p_{1}\right\}, \mathrm{Q}=0$. Then, the basic adaptive procedure is given by:

1: while subs $\neq \emptyset$ do

2: $\quad[\mathrm{Q} 1$, subs, $\mathrm{p}]=$ hprefine $\left(f\right.$, subs, $\left.\mathrm{p}, p_{\max }, \tau\right)$;

3: $\quad \mathrm{Q}=\mathrm{Q}+\mathrm{Q} 1$;

4: end while

5: Output Q.

Here, hprefine is a function, whose purpose is to identify those subintervals in subs, which need to be refined further for a sufficiently accurate approximation of the unknown integral. In addition, it outputs a set of subintervals (again denoted by subs), as well as an associated order vector (again denoted by $\mathrm{p}$ ) which result from applying the most appropriate refinement, i.e., either $h$ - or $p$-refinement as outlined in (i) and (ii) in Section 2.1 above, for each subinterval. Furthermore, hpref ine returns the sum Q1 of all quadrature values corresponding to subintervals in the input set subs for which no further refinement is deemed necessary. The essential steps are summarised in Algorithm 1; here, $p_{\min }$ denotes the minimal number of quadrature points to be employed on any given subinterval.

\subsection{Practical Aspects}

In this section we discuss the practical issues involved in the implementation of the procedure described in Section 2.3 within a given computing environment.

\subsubsection{Gauss-Quadrature Rules}

In principle, the adaptive procedure presented in Section 2.3 allows for any variable order family of quadrature rules to be exploited. For simplicity, in our numerical experiments presented in Section 2.5 below, we propose the use of (families 


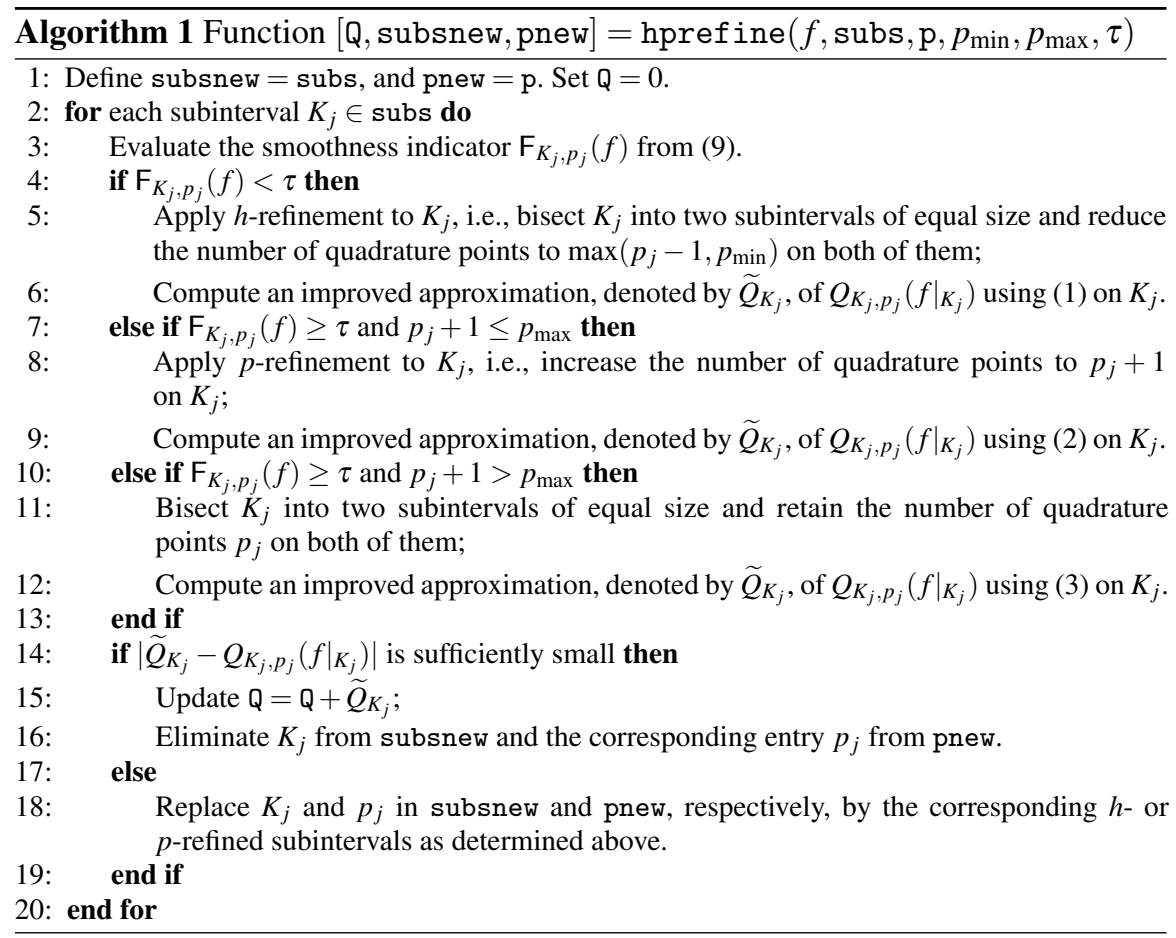

of) Gauss-type quadrature schemes. We emphasise, however, that more traditional schemes, including, for example, (fixed-order) Gauss-Kronrod or Clenshaw-Curtis rules, which are naturally hierarchical, may be employed as well (where the degree of exactness $2 p-2$ is desirable with regards to an accurate computation of the smoothness estimation, cf. Section 2.2). Incidentally, our numerical results indicate that, although non-hierarchical rules do not support the repeated use of all previously computed function evaluations, their potentially superior degree of accuracy, compared to their embedded counterparts, can be exploited very favourably within the $h p$-setting. Indeed, it is a well-known feature of $h p$-methods that they are particularly effective on a variable high-order level, cf., e.g., [17,18].

In the current article we employ Gauss-Legendre quadrature points and weights (with at least $p_{\min }=2$ points and weights); these quantities can be precomputed up to any given order $p_{\max }$ (in practice $p_{\max }=15$ is usually more than sufficient) or even be generated on the spot in an efficient way (see, e.g., [1]) if an upper bound $p_{\max }$ cannot be fixed. In addition, we note that the Gauss-Legendre rule based on $p$ points has a degree of exactness of $2 p-1$, i.e., the smoothness indicators derived in Section 2.2 can be computed by means of the formula given in (8). For a given maximum number $p_{\max }$, we store the points and weights of the Gauss-Legendre rules (on the reference interval $[-1,1]$ ) with up to $p_{\max }$ points in two $p_{\max } \times\left(p_{\max }-1\right)$-matrices $\boldsymbol{X}$ and $\boldsymbol{W}$, respectively; here, for parameters $p=2, \ldots, p_{\max }$, the $p$-th columns of $\boldsymbol{X}$ and $\boldsymbol{W}$ are built from the points and 
weights of the corresponding $p$-point Gauss-Legendre quadrature rule, respectively (and complementing the remaining entries in all but the last column by zeros):

$$
\boldsymbol{X}=\left(\begin{array}{cccc}
\widehat{x}_{2,1} & \widehat{x}_{3,1} & \cdots & \widehat{x}_{p_{\max }, 1} \\
\widehat{x}_{2,2} & \vdots & & \\
\widehat{x}_{3,3} & & \vdots \\
\mathbf{0} & \ddots & \\
& & \widehat{x}_{p_{\max }, p_{\max }}
\end{array}\right), \quad \boldsymbol{W}=\left(\begin{array}{cccc}
w_{2,1} & w_{3,1} & \cdots & w_{p_{\max }, 1} \\
w_{2,2} & \vdots & & \\
w_{3,3} & & \vdots \\
& \mathbf{0} & \ddots & \\
& & & w_{p_{\max }, p_{\max }}
\end{array}\right)
$$

We note that, for other quadrature rules, the number of rows in the above matrices may be different.

\subsubsection{Vectorised Quadrature}

Following the ideas of [19] we use a vectorised quadrature implementation. This means that, instead of computing the integrals on the subintervals subs in Algorithm 1 one at a time, they are all computed at once. This can be accomplished by using fast vector- and matrix-operations, and by carrying out all necessary function evaluations in a single operation by computing the function to be integrated for a vector of input values. Specifically, we write the composite rule $I \approx \sum_{K_{i} \in \text { subs }} Q_{K_{i}, p_{i}}\left(\left.f\right|_{K_{i}}\right)=\sum_{K_{i} \in \text { subs }} h_{i} / 2 \sum_{k=1}^{p_{i}} w_{p_{i}, k}\left(f \circ \phi_{K_{i}}\right)\left(\widehat{x}_{p_{i}, k}\right)$ as a dot product of a weight vector $\boldsymbol{w}$ and a function vector $f(\boldsymbol{x})$; here, the former vector contains all (scaled) weights $\left\{\frac{1}{2} h_{i} w_{p_{i}, k}\right\}_{i, k}$, and the latter vector represents the evaluation of the integrand function $f$ on the vector $\boldsymbol{x}$ of all corresponding quadrature points $\left\{\phi_{K_{i}}\left(\widehat{x}_{p_{i}, k}\right)\right\}_{i, k}$ appearing in the sum above. Evidently, these vectors can be built efficiently by extracting (and affinely mapping and scaling) the corresponding rows from the matrices $\boldsymbol{X}$ and $\boldsymbol{W}$ in (10). We emphasise that applying vectorised quadrature crucially improves the performance of the overall adaptive procedure (provided that such a technology is available in a given computing environment).

\subsubsection{Smoothness Estimators}

As already noted, computing the smoothness indicators from (8) does not need any additional function evaluations of the function $f$; they only require the values of the Legendre polynomials $\widehat{L}_{p-1}$ and $\widehat{L}_{p-2}$ at the points $\left\{\widehat{x}_{p, k}\right\}_{k=1}^{p}$, for $p=2, \ldots, p_{\max }$. These quantities are again precomputable, and can be stored in two matrices 


$$
\boldsymbol{L}_{1}=\left(\begin{array}{cccc}
L_{1}\left(\widehat{x}_{2,1}\right) & L_{2}\left(\widehat{x}_{3,1}\right) & \cdots & L_{p_{\max }-1}\left(\widehat{x}_{p_{\max }, 1}\right) \\
L_{1}\left(\widehat{x}_{2,2}\right) & \vdots & & \\
L_{2}\left(\widehat{x}_{3,3}\right) & & \vdots \\
& \mathbf{0} & \ddots & \\
& & & L_{p_{\max }-1}\left(\widehat{x}_{p_{\max }, p_{\max }}\right)
\end{array}\right)
$$

and

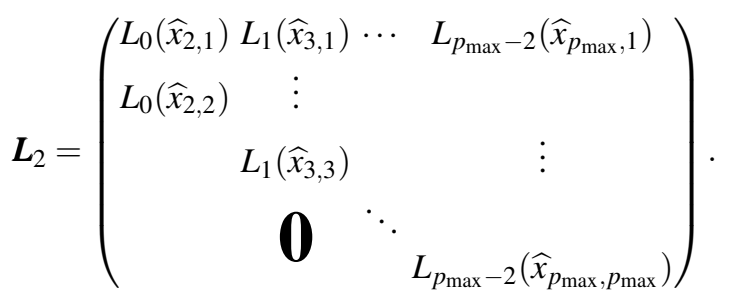

Then, the sums in (8) are vectorised similarly as described above. In particular, the computation of the smoothness estimators can be undertaken with an almost negligible computational cost.

\subsubsection{Stopping Criterion}

In order to implement the stopping-type criterion in line 14 of Algorithm 1, we exploit an idea that was proposed in the context of adaptive Simpson quadrature in [7]. More precisely, given a possibly rough approximation iguess $\approx \int_{a}^{b} f(x) \mathrm{d} x$ of the unknown integral $I$ (e.g., obtained from a Monte-Carlo calculation such that both the approximation and the exact value are of the same magnitude; cf. [7]), and a tolerance tol $>0$, we redefine iguess $=$ iguess $*$ tol/eps; here, eps represents the smallest (positive) machine number in a given computing environment. Then, using the comparison operator $==$, we accept the difference $\left|\widetilde{Q}_{K_{j}}-Q_{K_{j}, p_{j}}\left(\left.f\right|_{K_{j}}\right)\right|$ to be sufficiently small with respect to the given tolerance tol if the logical call iguess $+\left|\widetilde{Q}_{K_{j}}-Q_{K_{j}, p_{j}}\left(\left.f\right|_{K_{j}}\right)\right|==$ iguess; yields a true value. In this way tol represents a reasonable approximation of the relative error.

\subsection{Numerical Examples}

In order to test our approach, we consider a number of benchmark problems on the interval $[0,1]$. Specifically, the following functions will be studied: 


\begin{tabular}{rrr}
\hline & hp-adaptive quadrature & adaptive Simpson quadrature \\
\hline$f_{1}$ & 52 & 4,096 \\
$f_{2}$ & 1,718 & 25,488 \\
$f_{3}$ & 2,427 & 72,528 \\
$f_{4}$ & 21,005 & $1,213,680$ \\
$f_{5}$ & 1,493 & 784 \\
\hline
\end{tabular}

Table 1 Number of function evaluations for $h p$-type adaptive quadrature and adaptive Simpson quadrature.

$$
\begin{aligned}
f_{1}(x)= & \exp (x), \\
f_{2}(x)= & |x-1 / 3|^{1 / 2}, \\
f_{3}(x)= & \operatorname{sech}(10(x-1 / 5))^{2}+\operatorname{sech}(100(x-2 / 5))^{4} \\
& +\operatorname{sech}(1000(x-3 / 5))^{6}+\operatorname{sech}(1000(x-4 / 5))^{8}, \\
f_{4}(x)= & \cos \left(1000 x^{2}\right), \\
f_{5}(x)= & \begin{cases}0 & \text { if } x<1 / 3 \\
1 & \text { if } x \geq 1 / 3 .\end{cases}
\end{aligned}
$$

Whilst the first function, $f_{1}$, is analytic, $f_{2}$ is smooth except at $1 / 3$ (see Figure 1). Furthermore, $f_{3}$ was proposed in [9] in the context of the CHEBFUN package [10]; this is a smooth function that exhibits several very thin spikes (see Figure 2). Moreover, $f_{4}$ is highly oscillating towards the right end point 1 , and $f_{5}$ is an example of a discontinuous function.

We perform our computations in MATLAB, and set the tolerance to tol $=$ $0.3 \times 10^{-15}$ (which is close to machine precision in MATLAB), the smoothness estimation parameter is prescribed as $\tau=0.6, p_{\min }=2$, and $p_{\max }=15$. Within this setting, our adaptive procedure generates results that are accurate to machine precision, for all of the considered examples. In Table 1, for each of the functions $f_{1}, \ldots, f_{5}$ above, we present the number of function evaluations (counting an evaluation of the given function $f_{i}$ for a vector-valued argument $\boldsymbol{x}=\left(x_{1}, \ldots, x_{n}\right)$, i.e., $f_{i}(\boldsymbol{x})=\left(f_{i}\left(x_{1}\right), \ldots, f_{i}\left(x_{n}\right)\right)$, as $\left.n\right)$ for the proposed $h p$-adaptive quadrature procedure, as well as the corresponding number for a classical adaptive Simpson method from [7] (which is based on employing the two end points, as well as the midpoint on each subinterval, and reuses the former two points without recomputing), with the same tolerance value tol $=0.3 \times 10^{-15}$. Except for the last function, $f_{5}$, where a low-order quadrature rule is more effective, the remarkable efficiency of the proposed $h p$-type quadrature becomes clearly visible.

In order to illustrate how the $h p$-adaptive procedure performs, we depict the final $h p$-mesh for $f_{2}$ and $f_{3}$ in Figure 1 and Figure 2, respectively. Here, along the horizontal axis we present the subintervals obtained as a result of the adaptive process, and on the vertical axis the number of quadrature points introduced on each subinterval is displayed. In both examples, we see that smooth regions in the un- 

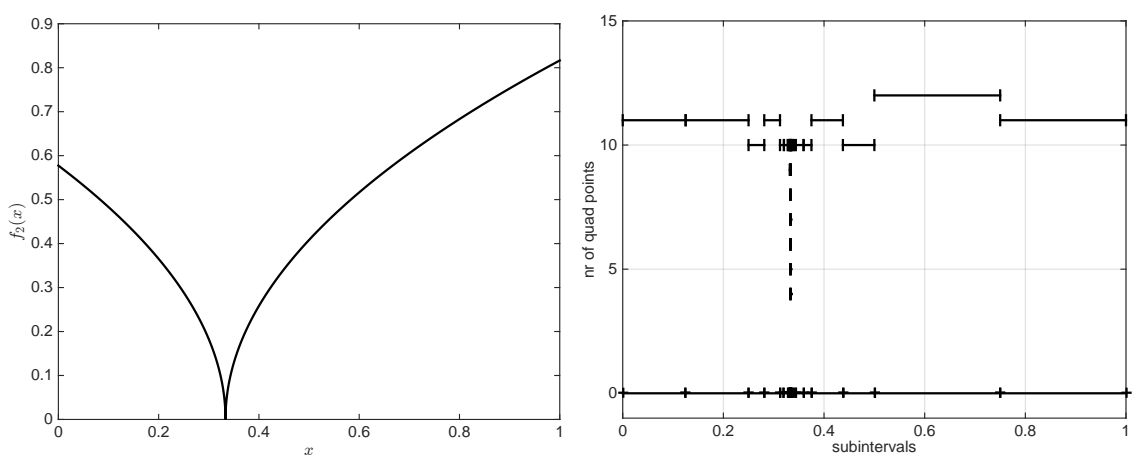

Fig. 1 Function $f_{2}$ : Graph (left) and $h p$-mesh (right).
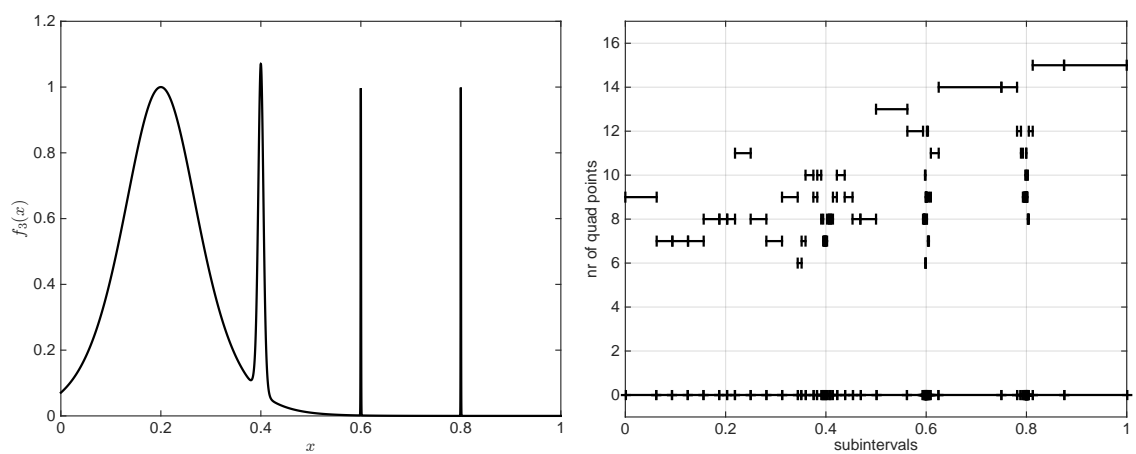

Fig. 2 Function $f_{3}$ : Graph (left) and $h p$-mesh (right).

derlying integrand are resolved by employing larger subintervals featuring a higher number of quadrature points, whereas close to singularities, the number of quadrature points is kept low on very small integration subdomains. It is noteworthy that this behaviour is well-known from $h p$-FEMs for differential equations, where highorder algebraic or even exponential convergence rates can be obtained by applying this type of $h p$-refinement procedure; see [18] for details.

\section{Conclusions}

In this article we proposed a new adaptive quadrature strategy, which features both local subdivision of the integration domain, as well as local variation of the number of quadrature points employed on each subinterval. Our approach is inspired by the $h p$-adaptive FEM methodology based on $h p$-adaptive smoothness testing. In combination with a vectorised quadrature implementation, the proposed adaptive quadrature algorithm is able to deliver highly accurate results in a very efficient manner. 
Since our approach is closely related to the $h p$-FEM technique, it can be extended to multiple dimensions, including, in particular, the application of anisotropic refinements of the underlying domain of integration, together with the exploitation of different numbers of quadrature points in each coordinate direction on each subinterval (based, for example, on anisotropic Sobolev embeddings as outlined in $[6, \S 3.1]$ ).

\section{References}

1. I. Bogaert, Iteration-free computation of Gauss-Legendre quadrature nodes and weights, SIAM J. Sci. Comput. 36 (2014), no. 3, A1008-A1026. MR 3209728

2. G. Dahlquist and Å. Björck, Numerical methods in scientific computing. Vol. I, Society for Industrial and Applied Mathematics (SIAM), Philadelphia, PA, 2008.

3. P. J. Davis and P. Rabinowitz, Methods of numerical integration, Dover Publications, Inc., Mineola, NY, 2007, Corrected reprint of the second (1984) edition.

4. L. Demkowicz, Computing with hp-adaptive finite elements. Vol. 1, Chapman \& Hall/CRC Applied Mathematics and Nonlinear Science Series, Chapman \& Hall/CRC, Boca Raton, FL, 2007, One and two dimensional elliptic and Maxwell problems.

5. R. DeVore and K. Scherer, Variable knot, variable degree spline approximation to $x^{\beta}$, Quantitative approximation (Proc. Internat. Sympos., Bonn, 1979), Academic Press, New YorkLondon, 1980, pp. 121-131.

6. T. Fankhauser, T. P. Wihler, and M. Wirz, The hp-adaptive FEM based on continuous Sobolev embeddings: isotropic refinements, Comput. Math. Appl. 67 (2014), no. 4, 854-868.

7. W. Gander and W. Gautschi, Adaptive quadrature-revisited, BIT 40 (2000), no. 1, 84-101.

8. W. Gui and I. Babuška, The $h, p$ and $h-p$ versions of the finite element method in onedimension, parts I-III, Numer. Math. 49 (1986), no. 6, 577-683.

9. N. Hale, Spike integral, 2010, http://www.chebfun.org/examples/quad/SpikeIntegral.html.

10. N. Hale and L. N. Trefethen, Chebfun and numerical quadrature, Sci. China Math. 55 (2012), no. $9,1749-1760$.

11. P. Houston and E. Süli, A note on the design of $h p$-adaptive finite element methods for elliptic partial differential equations, Comput. Methods Appl. Mech. Engrg. 194(2-5) (2005), 229 243.

12. J. M. Melenk and B. I. Wohlmuth, On residual-based a posteriori error estimation in hp-FEM, Adv. Comp. Math. 15 (2001), 311-331.

13. W. F. Mitchell and M. A. McClain, A comparison of hp-adaptive strategies for elliptic partial differential equations, ACM Trans. Math. Softw. 41 (2014), 2:1-2:39.

14. W. H. Press, S. A. Teukolsky, W. T. Vetterling, and B. P. Flannery, Numerical recipes, third ed., Cambridge University Press, Cambridge, 2007, The art of scientific computing.

15. K. Scherer, On optimal global error bounds obtained by scaled local error estimates, Numer. Math. 36 (1980/81), no. 2, 151-176.

16. D. Schötzau, C. Schwab, and T. P. Wihler, hp-DGFEM for second-order mixed elliptic problems in polyhedra, Math. Comp. (in press).

17. C. Schwab, Variable order composite quadrature of singular and nearly singular integrals, Computing 53 (1994), no. 2, 173-194. MR 1300776 (96a:65035)

18. C. Schwab, p-and hp-FEM - Theory and application to solid and fluid mechanics, Oxford University Press, Oxford, 1998.

19. L. F. Shampine, Vectorized adaptive quadrature in Matlab, J. Comput. Appl. Math. 211 (2008), no. 2, 131-140.

20. P. Solin, K. Segeth, and I. Dolezel, Higher-order finite element methods, Studies in advanced mathematics, Chapman \& Hall/CRC, Boca Raton, London, 2004.

21. T. P. Wihler, An hp-adaptive strategy based on continuous Sobolev embeddings, J. Comput. Appl. Math. 235 (2011), 2731-2739. 